\title{
Beam Steering Investigation on Multiple Antenna System using Radiation Pattern Reconfigurable Array
}

\author{
Jaya Bharath \\ Advanced Communication Engineering \\ (ACE), Centre of Excellence, \\ Faculty of Electronic Engineering \\ Technology \\ Universiti Malaysia Perlis (UniMAP) \\ Arau, Perlis Malaysia \\ jbharath04@yahoo.com \\ Hasliza A Rahim \\ Advanced Communication Engineering \\ (ACE), Centre of Excellence, \\ Faculty of Electronic Engineering \\ Technology \\ Universiti Malaysia Perlis (UniMAP) \\ Arau, Perlis Malaysia \\ haslizarahim@unimap.edu.my
}

\author{
Thennarasan Sabapathy \\ Advanced Communication Engineering \\ (ACE), Centre of Excellence, \\ Faculty of Electronic Engineering \\ Technology \\ Universiti Malaysia Perlis (UniMAP) \\ Arau, Perlis Malaysia \\ thennarasan@unimap.edu.my \\ Muhammad Ramlee Kamarudin \\ Faculty of Electrical and Electronic \\ Engineering \\ Universiti Tun Hussein Onn Malaysia \\ (UTHM) \\ Batu Pahat, Johor, Malaysia \\ mramlee@uthm.edu.my
}

\author{
Muzammil Jusoh \\ Advanced Communication Engineering \\ $(A C E)$, Centre of Excellence, \\ Faculty of Electronic Engineering \\ Technology
}

Universiti Malaysia Perlis (UniMAP)

Arau, Perlis Malaysia

muzammil@unimap.edu.my

Ping Jack Soh

Centre for Wireless Communications (CWC)

University of Oulu

Finland

pingjack.soh@oulu.fi

\begin{abstract}
In this research, a radiation pattern reconfigurable (RPR) based multiple antenna was designed using a compact combination of a driven element and parasitic elements that operates at $3.5 \mathrm{GHz}$. The proposed antenna has the ability to change the radiation behavior to serve the user with better performance. The radiation characteristic of the antenna, especially the tilt angle could be reconfigured with the use of RF switches. The multiple antenna was designed on 94.4 mm $\times 110 \mathrm{~mm}$ Rogers substrate. First, an RPR antenna that uses parasitic patch array antenna was designed as a single element. Then this RPR was deployed as $2 \times 1$ RPR MIMO antenna using two types of configurations. Finally, the analysis showed that the multiple antenna arrangement using configuration II provides better results in terms of steering angle and gain.
\end{abstract}

Keywords - Antenna and Propagation, Reconfigurable Antenna, MIMO antenna

\section{INTRODUCTION}

Several researchers have been carried out research on the reconfigurable antenna for the past few years. Many wireless communication systems, such as radio cognitive systems, smart weapon systems, and wireless network systems get the benefit from the flexibility provided by reconfigurable antennas. The antenna that is capable to adjust the radiation pattern is addressed in the research world as a radiation pattern reconfigurable (RPR) antenna. This antenna is typically constructed using movable/rotatable structures or parasitic elements that are switchable and reactively loaded. Besides that, radiation pattern reconfigurable antenna also provides a simple solution in the beam steering application where it will lead to use least number of antenna elements with switching elements instead of expensive and complex structure [1][2]. To achieve the reconfigurability, the RF switches such as PIN-diode, varactor and MEMS, are widely implemented to achieve the different directions of the pattern towards the desired user [3][4][5]. Among them PIN diode switch is known for its fast switching response. The working concept of a PIN diode is that when it is forward biased, it is usually supplied by approximately $1 \mathrm{~mA}$ current and keeps the diode at ON state. Meanwhile, the PIN diode has a low capacitance when reverse biased, which prevents the RF signal from passing. As a result, it is in the OFF state thus serves as an insulator.

When compared to single antenna/channel communication, multiple input-multiple output (MIMO) is one of the most effective strategies to increase communication capacity. The combination of RPR antenna in MIMO technology is expected to give an advantage in terms of capacity. Reconfigurable antennas based on MIMO have been proposed by many researchers [6][3][7] to fulfil the increased capacity demand. In this research, the RPR antenna was implemented as multiple antenna or MIMO to increase the capability of the MIMO system [5]. Although various researches have been implementing the reconfigurable antenna with MIMO, limited works have been conducted research on radiation pattern reconfigurable antenna for MIMO application. Most of them focused on the user/mobile terminal [3][5]. Moreover, no pure pattern reconfiguration antenna has been designed that could have multiple steering directions before such antenna is deployed as an MIMO antenna.

Therefore, this research was conducted to carefully analyse and investigate the effect of beam steering that is available in RPR antenna if deployed as MIMO, specifically at mini base station or portable access point. A parasitic patch array antenna that adopted the Yagi-Uda principle [8] was used to model the RPR in this investigation. The RPRMIMO was investigated using $2 \times 1$ MIMO array with two kinds of configuration. A detailed description of the RPR antenna design and RPR-MIMO configurations are presented in the following section. Then a comprehensive investigation was conducted using RPR-MIMO and the results were presented in terms of S-parameter and polar radiation pattern. 


\section{RPR-MIMO CONFIGURATION AND ANALYSIS}

\section{A. Radiation Pattern Reconfigurable (RPR) Antenna}

Fig. 1 illustrated the physical structure of the patch array antenna. The antenna consists of three parallel patches which includes the single driven element at the centre and two parasitics at the left and right of the driven element. YagiUda principle was adopted in designing the RPR antenna, where a comprehensive background on this technique could be found in [9][2][10][11]. The antenna was designed in a full grounded Rogers dielectric substrate with a thickness of $1.524 \mathrm{~mm}$ and a dielectric constant $\left(\varepsilon_{\mathrm{r}}\right)$ of 3.55 . The loss tangent of the dielectric is 0.0027 . The driven element or the centre rectangular patch has a width of $X_{p} \mathrm{~mm}$ and a length of $Y_{p} \mathrm{~mm}$. The width and length were first calculated using the rectangular patch fundamental equations provided in [12] for center frequency of $3.5 \mathrm{GHz}$. The antenna was fed through the subminiature SMA probe from the back of the antenna. The feed location $a$, was optimized to achieve a desired input impedance. Besides that, the parasitic elements are smaller with the respect to the driven element where the width and length are labelled as $X p^{\prime}$ and $Y p^{\prime}$ respectively. Each parasitic element was connected to the ground plane at the back of the antenna through two artificial switches, where shorting pin (via) represent the artificial switch. The location of the switch was crucial in determining the optimum tilt angle. Two switches were needed in each parasitic to ensure the desired beam steering performance is achieved. SW1 and SW4 were located at $(-x 1,-y 1)$ and $(x 1$, $y 1)$ respectively to ensure the highest tilt angle, which is $\pm 31^{\circ}$. On the other hand, SW3 and SW4 were required to obtain the center beam or default directive beam at $0^{\circ}$ with the highest gain. Both parasitic elements are physically the same electrical length, switching their states between shortand open-circuited results in a variation of the elements electrical length. Thus, when the switch/shorting pin is short to the ground (ON state, via is active), it acts as a reflector (R). In contrast, when the switch is open (OFF state, via is inactive) it acts as a director (D). For instance, when SW is at ON state and the rest of the switches are at OFF state, it makes the parasitic element 1 operates as a reflector since it is connected to the ground plane. In contrast, parasitic element 2 operates as the director as the vias are inactive. Thus, this will produce the beam tilt angle of $+31^{\circ}$. The locations of the switches were optimized to achieve optimum beam tilt angle and common $S_{11}$ bandwidth at all beamsteered directions.

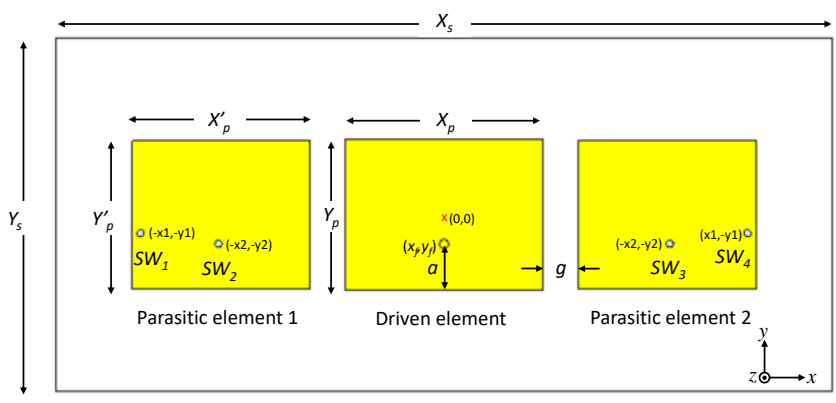

Fig. 1. Physical Array of Patch Array Antenna.

\section{B. RPR Antenna Deployed as Multiple Antenna}

Very few works have investigated the deployment of RPR antenna as multiple antenna or MIMO. In this work, $2 \mathrm{x}$ 1 configuration of RPR-MIMO was studied. The RPR was deployed in two configurations as shown in Fig. 2. In the first configuration, the RPR antenna was arranged using translate method and in the second combination of the MIMO, the RPR antenna was arranged by using mirror method. The edge-to-edge gap between the antenna was fixed where it was set to $\lambda_{\mathrm{g}} / 2$ based on $3.5 \mathrm{GHz}$ center frequency. Due to this, the distance between the feeders of the RPR antennas in Configuration 1 is greater than the distance between RPR antennas in Configuration 2. In other words, Gap $1>$ Gap 2. Both of the MIMO antennas were modeled on a substrate size of $94.4 \mathrm{~mm} \times 110 \mathrm{~mm}$.
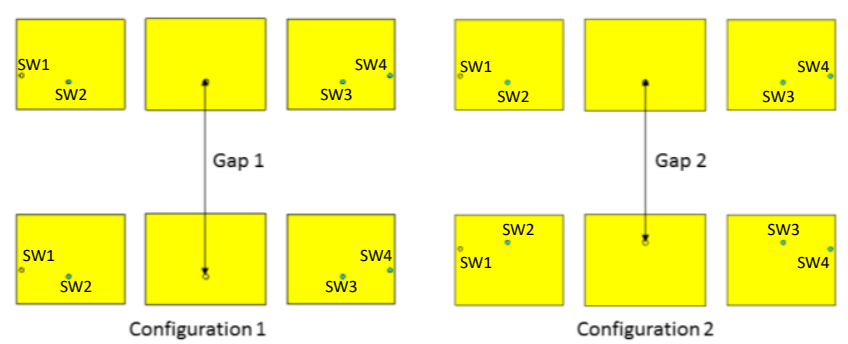

Fig. 2. Configuration of RPR-MIMO Antenna.

TABLE I. ANTENNA PHYSICAL DIMENSION

\begin{tabular}{|c|c|}
\hline Antenna Parameter & Measurement (mm) \\
\hline$X_{p}$ & 28 \\
\hline$Y_{p}$ & 21.40 \\
\hline$X_{p}^{\prime}$ & 25.20 \\
\hline$Y_{p}^{\prime}$ & 20.97 \\
\hline$g$ & 5 \\
\hline$X_{s}$ & 110 \\
\hline$Y_{s}$ & 50 \\
\hline$a$ & 6.6 \\
\hline$g$ & 0.98 \\
\hline$x_{f}$ & 0 \\
\hline$y_{f}$ & -4.1 \\
\hline$x 1$ & 43 \\
\hline$x 2$ & 32 \\
\hline$y 1$ & 2.6 \\
\hline$y 2$ & 4.1 \\
\hline Gap 1 (Conf 1) & 45.40 \\
\hline Gap 2 (Conf 2) & 37.20 \\
\hline
\end{tabular}

The performance difference between these two MIMO configurations was investigated and explained in the result section for a better understanding. Meanwhile as mentioned before, the size of the parasitic element plays a vital role in this investigation. To be more precise the size of the two parasitic elements is optimized to investigate the relationship between the size of parasitic element and the tilt angle of the main beam according to serve the user in a selective direction. 
Once determine the ideal size of the parasitic element which makes the main beam tilt more, then that size is kept and used for further investigation. Therefore the ratio of the parasitic element is fixed at $0.98 \%$ compared to the driven element. Apart from this, the switches locations are also crucial in determining the desired beam patterns with acceptable reflection coefficient results. All the related parameters of the antenna dimension are tabulated in Table I.

\section{RESULTS AND ANALYSIS}

This section presents simulated results of the RPR antenna in terms of S-parameter and radiation pattern results. The main focus of this research is to investigate the radiation characteristic of the RPR when implemented as RPR-MIMO.

\section{A. Results of RPR Antenna}

First, the operation principle and results of a single RPR antenna is presented. Table II presents the switching conditions and the respective beam steering. The presented switching configurations attain the optimum result in terms of tilt angle and gain. Without SW2 and SW3, the tilt angle at 0 was unable to attain high gain, where the gain was approximately $6 \mathrm{dBi}$. Therefore, additionally, SW2 and SW4 were needed. The respective beam pattern is depicted in Fig. 3. The polar radiation pattern shows that three sets of beam patterns that are almost identical in terms of shape and gain could be attained.

TABLE II. BEAMFORMING CHARACTERISTICS BASED ON SWITCHING CONDITIONS

\begin{tabular}{|c|c|c|c|c|c|}
\hline \multicolumn{4}{|c|}{ Switching Conditions } & \multirow{2}{*}{ Tilt angle } & \multirow{2}{*}{$\begin{array}{l}\text { Gain } \\
\text { (dBi) }\end{array}$} \\
\hline SW1 & SW2 & SW3 & SW4 & & \\
\hline 0 & 0 & 0 & 1 & $-31^{\circ}$ & 7.38 \\
\hline 1 & 0 & 0 & 0 & $+31^{\circ}$ & 7.38 \\
\hline 0 & 1 & 1 & 0 & $0^{\circ}$ & 7.42 \\
\hline
\end{tabular}

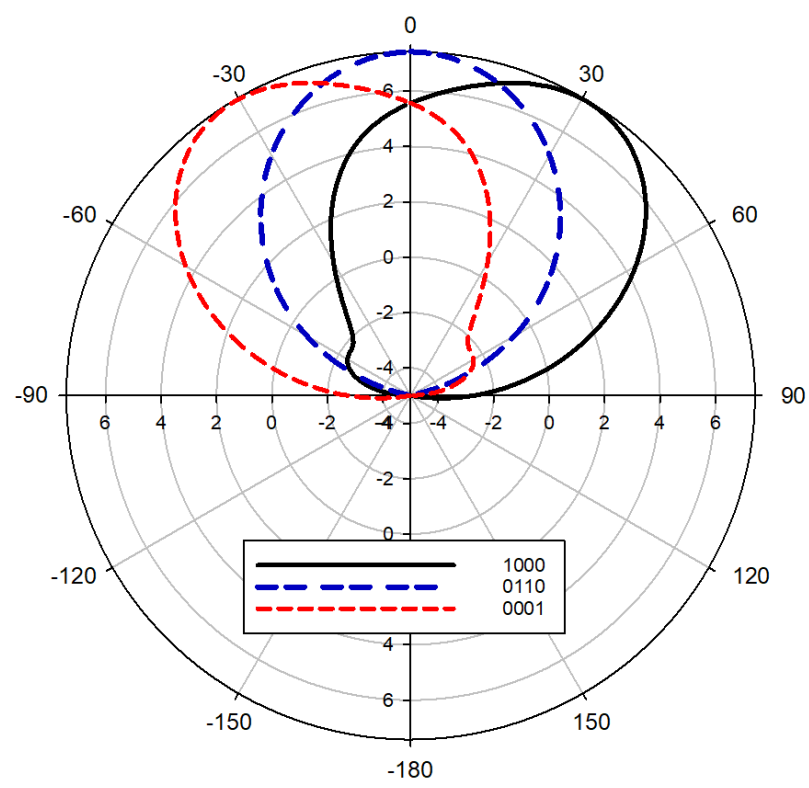

Fig. 3. The polar radiation pattern of RRP antenna at three switching conditions
Fig. 4 shows the reflection coefficient results for the RPR antenna. It can be noticed that the common $S_{11}$ bandwidth for all switching conditions is from $3.452 \mathrm{GHz}$ to $3.525 \mathrm{GHz}$ where approximately $73 \mathrm{MHz}$ bandwidth is attainable. Note that, 1000 and 0001 conditions produced similar results due to the symmetric location of the ON switches

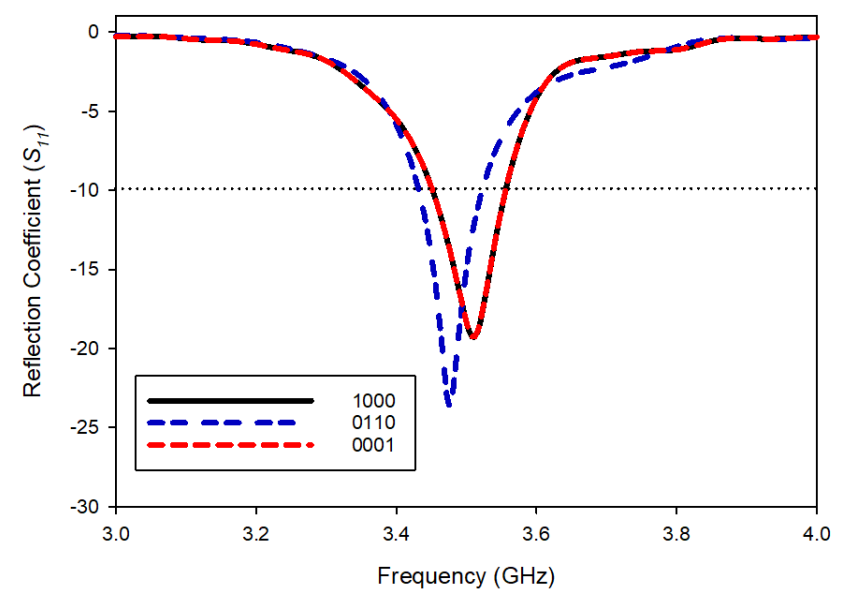

Fig. 4. The reflection coefficient of RRP antenna at three switching conditions.

\section{B. Results of RPR MIMO Antenna}

Based on Configuration 1, there are two sets of RPR antennas were placed in the opposite direction. One of the antenna is consists of one driven element at the centre and two parasitic elements at both sides of the driven element. The parasitic elements act either as a reflector/director and they are tasked to push the radiation pattern in both left and right direction based on the $\mathrm{ON}$ and $\mathrm{OFF}$ switch configuration.

This working principle is applied in Configuration 2 as well. In addition to that, the difference between Configuration 1 and Configuration 2 is the distance between the feeder which is identified as a Gap 1 and Gap 2. Simulations were carried out for all possible switching conditions of the RPR MIMO antenna. From Table 2, when the switching conditions were applied to RPR MIMO, nine possible sets of steering combinations were obtained. This work specifically focuses on the investigation of beam steering performance when the RPR is deployed as MIMO. However, the S-parameter results were also observed to see any significant changes. Fig. 5 shows the S-parameter results of the RPR MIMO for both configurations at all possible switching conditions.

For certain switching conditions and configuration, the resonant is slightly shifted from $3.5 \mathrm{GHz}$ frequency. However, it can be noticed that regardless the switching conditions and type of configuration, the $S$ parameter results are not much affected. The $S_{11}$ and $S_{22}$ results are less than $10 \mathrm{~dB}$ at $3.5 \mathrm{GHz}$. Meanwhile, the $S_{12}$ and $S_{22}$ results are approximately less than $-18 \mathrm{~dB}$. The main interest of this investigation is on the steering performance of the RPRMIMO. From Fig. 6, it can be seen that the tilt angle is largely affected for configuration 1 . 
The related steering angle and gain for each configuration are tabulated in Table III. For instance, at switching condition 0001:0001, the antenna 1 steering angle is $+16^{\circ}$ for configuration I while $-22^{\circ}$ for configuration 2 . The desired steering angle for this condition should be $-30^{\circ}$. Therefore, it could be noticed that, configuration 1 gives significant error. Similar performance differences can be observed for most of the switching conditions. All the false steering directions produced by Configuration 1 were highlighted in red in Table III. RPR-MIMO with configuration 2 does not give any false steering direction although the steering angles are dropped compared to the actual steering angle produced by the single RPR antenna. Apart from that, the steered antenna gains are also slightly reduced for all switching conditions. Comparing both configurations, Configurations 2 outperforms Configuration 1 in all steering conditions.
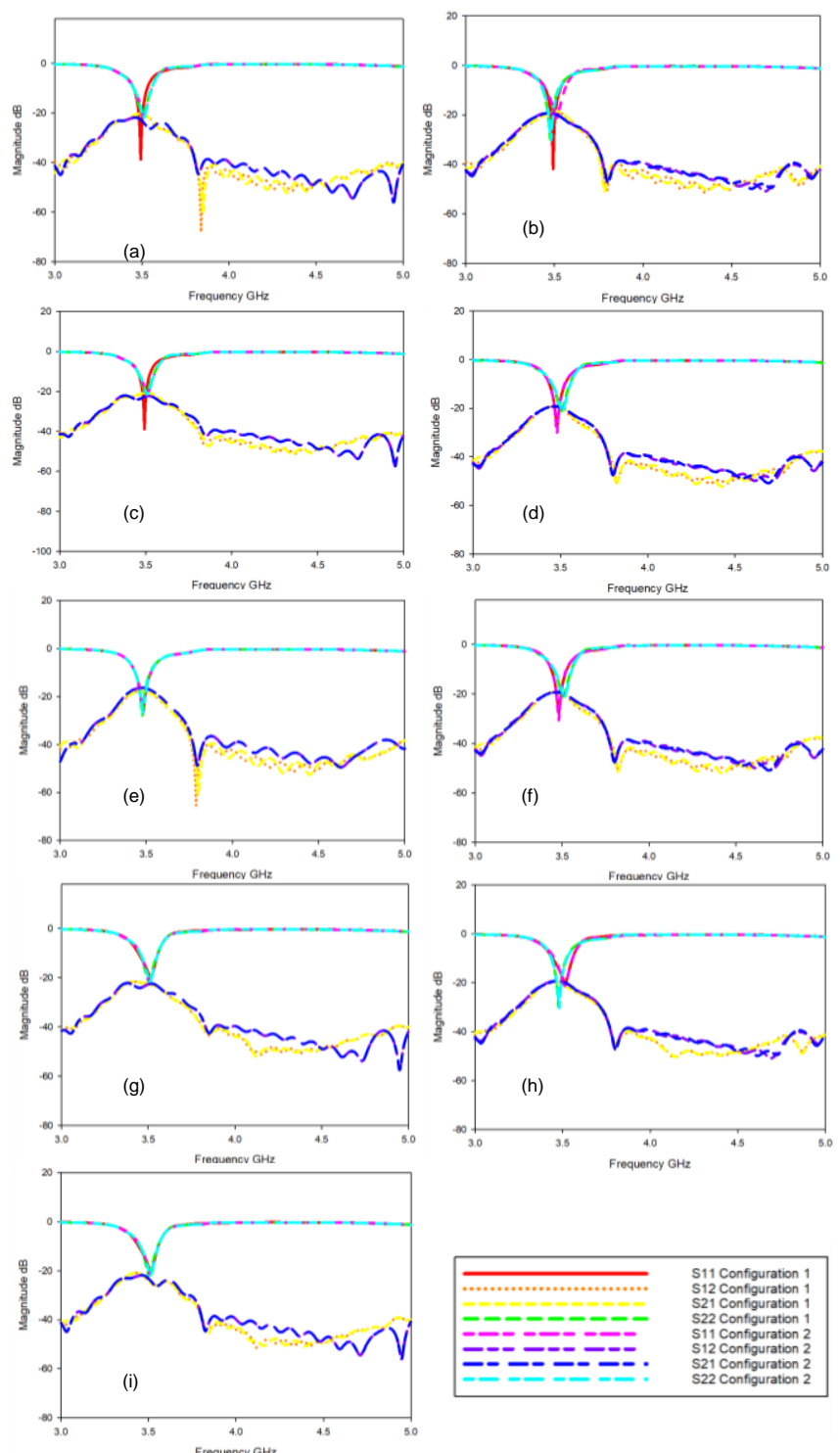

Fig. 5. S-parameter results for RPR MIMO using Configuration 1 and 2 for switching conditions: (a) 0001 0001, (b) 00010110 , (c) 0001 1000, (d) 0110 0001, (e) 0110 0110, (f) 0110 1000, (g) 1000 0001, (h) 10000110 and (i) 10001000 .
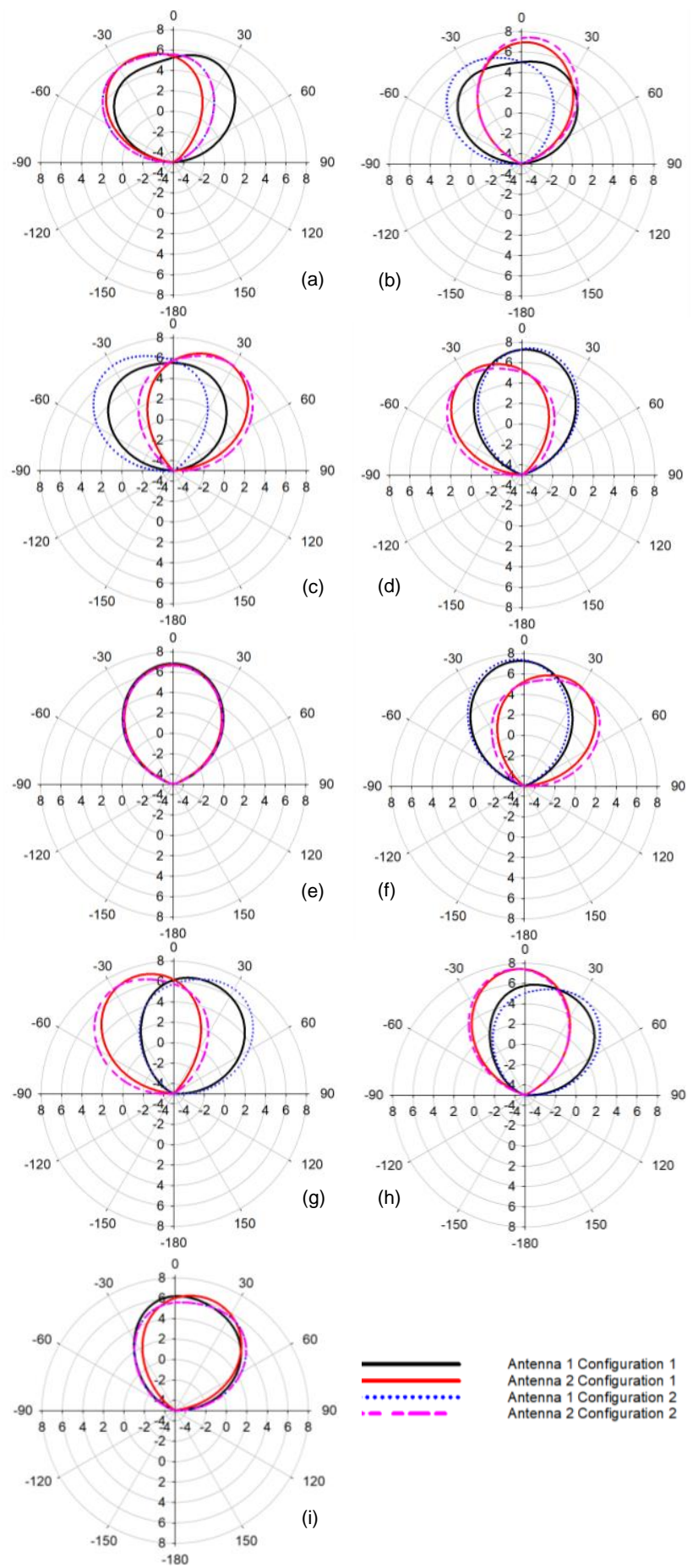

Fig. 6. Polar radiation pattern results for RPR MIMO using Configuration 1 and 2 for switching conditions: (a) 0001 0001, (b) 0001 0110, (c) 0001 1000, (d) 0110 0001, (e) 0110 0110, (f) 0110 1000, (g) 1000 0001, (h) 10000110 and (i) 10001000

\section{CONCLUSION}

This work primarily investigates the performance of RPR antenna when deployed as multiple antenna or MIMO. First, a single RPR antenna was designed to operate at $3.5 \mathrm{GHz}$ frequency with three directions, $\pm 31^{\circ}$ and $0^{\circ}$. Then the single RPR was arranged in form of $2 \times 1$ MIMO using two configurations, namely Configuration 1 and Configuration 2 . In configuration 1, the RPR antenna was translated while in Configuration 2 it was mirrored at $x$-axes. The investigation 
conducted has shown that the S-parameter results of RPRMIMO using both configurations were not much affected. However, the steering performance was slightly affected. Specifically, Configuration 1 yielded many false results. Although the steering angle was slightly dropped in Configuration 2, no false results were produced. To be more precise, when the RPR-MIMO operates under configuration 2 , it has a better chance to retain the beam steering characteristic of a single RPR antenna. In future work, the complete design of the proposed RPR multiple antenna system could be implemented with the DC biasing circuitry as the current work investigated the radiation mechanism using only proof concept switches.

TABLE III. BEAMFORMING CHARACTERISTICS BASED ON SWITCHING CONDITIONS FOR RPR-MIMO

\begin{tabular}{|c|c|c|c|c|c|c|c|c|}
\hline \multirow[t]{2}{*}{$\begin{array}{l}\text { Desired } \\
\text { Direction }\end{array}$} & \multicolumn{4}{|c|}{$\begin{array}{l}\text { Switching } \\
\text { Condition }\end{array}$} & \multicolumn{2}{|c|}{ Config 1} & \multicolumn{2}{|c|}{ Config 2} \\
\hline & $\begin{array}{l}S \\
1\end{array}$ & $\begin{array}{l}S \\
2\end{array}$ & $\begin{array}{l}S \\
3 \\
\end{array}$ & $\begin{array}{l}S \\
4\end{array}$ & $\theta$ & $\begin{array}{l}\text { Gain } \\
(\mathrm{dBi})\end{array}$ & $\theta$ & $\begin{array}{l}\text { Gain } \\
(\mathrm{dBi})\end{array}$ \\
\hline \multirow{2}{*}{$\begin{array}{l}\text { Ant } 1=-30^{\circ} \\
\text { Ant } 2=-30^{\circ}\end{array}$} & 0 & 0 & 0 & 1 & $+16^{\circ}$ & 5.74 & $-22^{\circ}$ & 5.74 \\
\hline & 0 & 0 & 0 & 1 & $-18^{\circ}$ & 5.92 & $-22^{\circ}$ & 5.74 \\
\hline \multirow{2}{*}{$\begin{array}{c}\text { Ant } 1=-30^{\circ} \\
\text { Ant } 2=0^{\circ}\end{array}$} & 0 & 0 & 0 & 1 & $+12^{\circ}$ & 5.16 & $-28^{\circ}$ & 6.09 \\
\hline & 0 & 1 & 1 & 0 & $-3^{\circ}$ & 6.96 & $-5^{\circ}$ & 7.45 \\
\hline \multirow{2}{*}{$\begin{array}{l}\text { Ant } 1=-30^{\circ} \\
\text { Ant } 2=+30^{\circ}\end{array}$} & 0 & 0 & 0 & 1 & $+10^{\circ}$ & 5.53 & $-26^{\circ}$ & 6.88 \\
\hline & 1 & 0 & 0 & 0 & $-22^{\circ}$ & 6.96 & $26^{\circ}$ & 6.88 \\
\hline \multirow{2}{*}{$\begin{array}{c}\text { Ant } 1=0^{\circ} \\
\text { Ant } 2=-30^{\circ}\end{array}$} & 0 & 1 & 1 & 0 & $-3^{\circ}$ & 7.29 & $-5^{\circ}$ & 7.45 \\
\hline & 0 & 0 & 0 & 1 & $-22^{\circ}$ & 6.36 & $-28^{\circ}$ & 6.09 \\
\hline \multirow{2}{*}{$\begin{array}{l}\text { Ant } 1=0^{\circ} \\
\text { Ant } 2=0^{\circ}\end{array}$} & 0 & 1 & 1 & 0 & $0^{\circ}$ & 6.85 & $0^{\circ}$ & 6.64 \\
\hline & 0 & 1 & 1 & 0 & 0 & 6.71 & 0 & 6.64 \\
\hline \multirow{2}{*}{$\begin{array}{c}\text { Ant } 1=0^{\circ} \\
\text { Ant } 2=+30^{\circ}\end{array}$} & 0 & 1 & 1 & 0 & $+3^{\circ}$ & 7.29 & $+5^{\circ}$ & 7.45 \\
\hline & 1 & 0 & 0 & 0 & $+22^{\circ}$ & 6.36 & $+28^{\circ}$ & 6.09 \\
\hline \multirow{2}{*}{$\begin{array}{l}\text { Ant } 1=+30^{\circ} \\
\text { Ant } 2=-30^{\circ}\end{array}$} & 1 & 0 & 0 & 0 & $+13^{\circ}$ & 6.52 & $+26^{\circ}$ & 6.88 \\
\hline & 0 & 0 & 0 & 1 & $-3^{\circ}$ & 6.96 & $-26^{\circ}$ & 6.88 \\
\hline \multirow{2}{*}{$\begin{array}{c}\text { Ant } 1=+30^{\circ} \\
\text { Ant } 2=0^{\circ}\end{array}$} & 1 & 0 & 0 & 0 & $+3^{\circ}$ & 7.44 & $+28^{\circ}$ & 6.09 \\
\hline & 0 & 1 & 1 & 0 & $-3^{\circ}$ & 6.96 & $+5^{\circ}$ & 7.45 \\
\hline \multirow{2}{*}{$\begin{array}{l}\text { Ant } 1=+30^{\circ} \\
\text { Ant } 2=+30^{\circ}\end{array}$} & 1 & 0 & 0 & 0 & $+2^{\circ}$ & 6.23 & $+22^{\circ}$ & 5.74 \\
\hline & 1 & 0 & 0 & 0 & $+14^{\circ}$ & 6.43 & $+22^{\circ}$ & 5.74 \\
\hline
\end{tabular}

\section{REFERENCES}

[1] K. Laafif, M. Bouslama, and A. Gharsallah, "Pattern Reconfigurable Antenna Design for for 5G mobile communication systems," pp. 13-15, 2017.

[2] T. Sabapathy, S. Member, R. B. Ahmad, S. Member, and M. Jusoh, "The Effect of Shorting Pin Locations on the Performance of a Pattern Reconfigurable Yagi-Uda Patch Antenna," pp. 0-3, 2014

[3] P. Fang, K. Wang, M. Wolfmüller, and T. F. Eibert, "Radiation Pattern Reconfigurable Antenna for MIMO Systems with Antenna Tuning Switches," pp. 503-504, 2018.

[4] Z. Nie, H. Zhai, L. Liu, J. Li, D. Hu, and J. Shi, “A Dual-Polarized Frequency-Reconfigurable Low-Profile Antenna with Harmonic Suppression for 5G Application," IEEE Antennas Wirel. Propag. Lett., vol. 18, no. 6, pp. 1228-1232, 2019, doi: 10.1109/LAWP.2019.2913170.

[5] S. Padmanathan, A. A. Al-hadi, P. J. Soh, M. F. Jamlos, and F. C. Seman, "Dual-Port $28 \mathrm{GHz}$ Pattern Reconfigurable Quadruple Parasitic IFA Design for MIMO 5G Mobile Terminal,” pp. 12-15.

[6] T. Alam, S. R. Thummaluru, and R. K. Chaudhary, "Two-Port MIMO Wide-Band Antenna With Two-Port MIMO Reconfigurable Antenna for Cognitive Radio Platforms," pp. 1215,2018

[7] W. Zhang, Z. Weng, and L. Wang, "Design of a Dual-band MIMO Antenna for 5G Smartphone Application,” pp. 11-13.

[8] X. S. Yang, B. Z. Wang, and H. L. Liu, "Reconfigurable yagi patch array by utilizing Odd-even-mode method," J. Electromagn. Waves Appl., vol. 20, no. 13, pp. 1725-1738, 2006, doi: $10.1163 / 156939306779292282$.

[9] T. Sabapathy, M. F. Jamlos, R. B. Ahmad, M. Jusoh, and M. I. Jais, "A reconfigurable microstrip rectangular parasitic array antenna," IEEE Symp. Wirel. Technol. Appl. ISWTA, pp. 372-375, 2013, doi: 10.1109/ISWTA.2013.6688807.

[10] T. Sabapathy, R. B. Ahmad, M. Jusoh, M. R. Kamarudin, and A. Alomainy, "A pattern-reconfigurable parasitic patch antenna using BAR and HPND PIN Diode," 8th Eur. Conf. Antennas Propagation, EuCAP 2014, no. EuCAP, pp. 3444-3445, 2014, doi: 10.1109/EuCAP.2014.6902569

[11] T. Sabapathy, M. Jusoh, R. B. Ahmad, and M. R. Kamarudin, "Wide angle scanning reconfigurable beam steering antenna," Eur. Microw. Week 2015 , pp. 1451-1454, 2015, doi: 10.1109/EuMC.2015.7346047.

[12] C. A. Balanis, "Balanis - Antenna Theory - 2nd edit.pdf.” 2016. 